ARTICLE

\title{
Large-Scale Computation of Welding Residual Stress
}

\author{
Akihiro KAWAGUCHI ${ }^{*}$, Shinsuke ITOH, \\ Masahito MOCHIZUKI and Masashi KAMEYAMA \\ Osaka University, 2-1 Yamada-oka, Suita-city, Osaka-fu, 565-0871, Japan
}

\begin{abstract}
This paper presents a technique for the speedup of welding residual stress analyses and an application for actual equipment. The iterative substructure method (ISM) developed by the authors rapidly carries out the finite element method (FEM) based on thermo-elasto-plastic theory. Generally, thermo-elasto-plastic analysis has required a huge amount of computational time. However, in this study, the technique reduces the computational time by computing the small region near the weld torch as a strong nonlinear region, and the remaining large area as an elastic linear region, so that only the stiffness matrix needs to be remade when the convergence is failed. The validity of this technique was verified as follows. The residual stress distribution and the angular deformation were not affected and the analysis was computed within a reasonable computational time. In addition, the analysis result of the residual stress agreed well with the experimental result. The weld joint in a pressurizer valve nozzle was analyzed as an application of a large-scale model of actual equipment. Using a three-dimensional cylindrical model of the nozzle with approximately one-hundred-thousand DOF, 70 passes of a multi-pass weld analysis were carried out. The residual stress of the multi-pass weld joint reached a high level that was close to the yield stress.
\end{abstract}

KEYWORDS: thermo-elastic-plastic analysis, finite element method, weld residual stress, large-scale computation, iterative substructure method

\section{Introduction}

Simulation technologies in research and development for new products reduce the costs and time for making a prototype and conducting experiments. Simulations can accelerate the introduction of products to market, and this capability is important to survive in today's keen global competition. Recently, analyses of not only simple models but also large or complicated structures have significantly progressed due to improved computer performance. Nevertheless, the analysis model for an automobile or ship has millions of degrees of freedom (DOF). In such cases, the model size is limited by the memory size and the analysis requires much computational time. Consequently, it is important to develop an analysis that is faster and uses less memory.

A welding analysis requires many calculation steps because of the sequential calculations between heating and cooling. Additionally, a welding analysis is a locally strong nonstationary and nonlinear problem, because of the high temperature in the area near the weld torch. Therefore, existing analysis methods are limited to models of test specimens that are tens of thousands DOF. Although various general-purpose software packages for welding analysis have been developed, they have the same limitations on the number of elements in the model and the computational time. ${ }^{1-6)}$ Therefore, a calculation technique applicable to large-scale welding problems with hundreds of thousands of DOF is needed for analyzing the welding problems of actual

*Corresponding author, E-mail:kawaguchi@mapse.eng.osaka-u.ac. jp

(C) 2011 Atomic Energy Society of Japan, All Rights Reserved. equipment.

The electrical energy generated by nuclear power accounts for one-third of the power needs in Japan. The construction of a nuclear power plant takes approximately 10 years, and long-term use must plan for repairs or exchanges of existing power plants. Recently, stress corrosion cracking (SCC) has occurred on the inner surfaces of welded dissimilar joints in reactor piping systems. SCC, which is the primary reason for the repair of nuclear pressure vessels, results from the joining of materials, the environment and tensile stress. As an example, one cause of SCC is the tensile residual stress after welding. Therefore, the prediction of the residual stress in a multi-pass weld joint of dissimilar materials is very important. However, the stress field of the multi-pass weld joint becomes complicated by the multiple heat histories, and measurement of stress field is difficult.

This paper proposes a technique to speed up the large-scale computation of the welding residual stress of a multi-pass weld joint of dissimilar materials. This technique is applied to a pressurizer relief valve nozzle damaged by SCC.

\section{Theory of the Iterative Substructure Method}

The welding phenomenon is a locally strong nonstationary and nonlinear problem. This section describes the iterative substructure method (ISM) developed by the authors to rapidly carry out the finite element method (FEM) based on thermo-elasto-plastic theory. ${ }^{7-14)}$

The welding phenomenon has two features. One is the local nonlinear region that is near the welding torch and is 
caused by the temperature dependence of the material property or plasticity, whereas most of the remaining area is nearly linear elastic. The other feature is that the strong nonlinear area moves with time as the torch moves.

Generally, welding simulations consume much computational time and memory to calculate the whole model as a nonlinear problem. Therefore, in the proposed technique, the whole area is divided into a strong nonlinear region $B$ and a weak nonlinear region $A$ by the border $\Gamma$, as shown in Fig. 1, and the calculation of each region can be done by a suitable method by ISM.

The approximation solution in FEM is solved by the following conditional expressions:

(1) Compatibility equation (strain-displacement relation)

(2) Constituent equation (stress-strain relation)

(3) Balanced equation (expression of equilibrium of force)

Region A and region B are separately solved by ISM. (1) The compatibility equation and (2) the constituent equation are able to meet within the $\mathrm{A}$ and $\mathrm{B}$ regions and border $\Gamma$ when using the conforming element, but (3) the balanced equation is not able to meet at border $\Gamma$. Therefore, (3) the balanced equation is found by iterative calculation of_(1) the compatibility equation and (2) the constituent equation.

In addition, the stiffness changes with the welding progress. The stiffness of the $\mathrm{B}$ region, which is the high-temperature area, changes significantly, but the stiffness of the A region, which is the low-temperature region, shows only negligible changes. Therefore, if the change of the stiffness of the A region is small, the stiffness of the previous step can be used. Then, the stiffness is regenerated with the convergent decrease of the calculation.

The flow is as follows:

(1) Defining regions A, B and border $\Gamma$ based on the temperature distribution and the yield state. The temperature distribution was used from temperature analysis result was carried out before ISM. The yield state was used from the result of the previous step.

(2) Solving the whole and calculating the stiffness matrix.

(3) Solving the whole and calculating the increment of the displacement at border $\Gamma$.

(4) Calculating the B region, which is a nonlinear problem based on the displacement of border $\Gamma$, as the forced

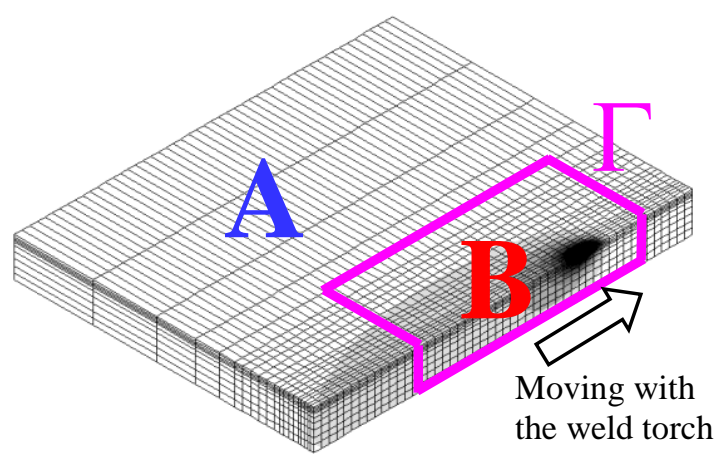

Fig. 1 Regions A, B and border $\Gamma$ used in ISM displacement.

(5) Solving the modified displacement by the residual force at border $\Gamma$.

(6) Iterating steps (1) - (5) until the calculation converges.

The whole stiffness matrix is not remade in the convergence calculation. This means the iterative calculations are only for the $\mathrm{B}$ region, which is the strong but small nonlinear region. In addition, if the changes of the whole stiffness matrix are negligible when the $\mathrm{B}$ region moves, the number of iterations to solve the large-scale simultaneous equation decreases by using the stiffness matrix in the previous step. As a result, it is an effective way to shorten the computational time. The flowchart of ISM is shown in Fig. 2.

In this study, the element with plastic constraints or with over $300{ }^{\circ} \mathrm{C}$ of the average temperature of nodes ware defined $B$ region. The calculated residual stress is not sensitive to the assumed location of the border $\Gamma$ because the static equilibrium is achieved by convergence check when the step is finished. The condition of the average temperature of nodes affects the computational time. The condition of high average temperature makes B region small and poorly converged. The low temperature makes $\mathrm{B}$ region big and to lengthen the computational time.

\section{Comparison of ISM with the Conventional Method}

This section compares ISM with the conventional method to test the effectiveness of ISM as a high-speed large-scale computational method. Specifically, the computational time for each method is compared. The residual stress of the welding direction and the angular distortion that are industry problems are examined. The calculations were carried out on the three-dimensional half-model of a welded plate, $100 \times$ $100 \times 6 \mathrm{~mm}$, with the traveling heat source at the center of the model, as shown in Fig. 3. The half-model was used to analyze a similar joint. The weld conditions were welding heat input $Q=252 \mathrm{~J} / \mathrm{mm}$, welding speed $v=2 \mathrm{~mm} / \mathrm{s}$. The material properties, which are shown in Fig. 4, are for the

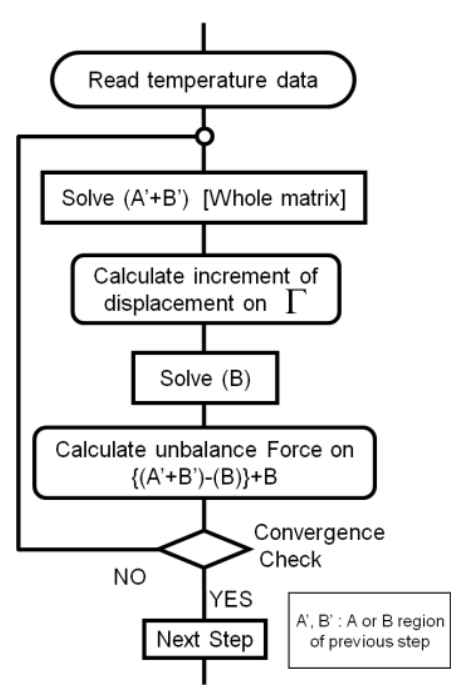

Fig. 2 The flowchart of ISM 


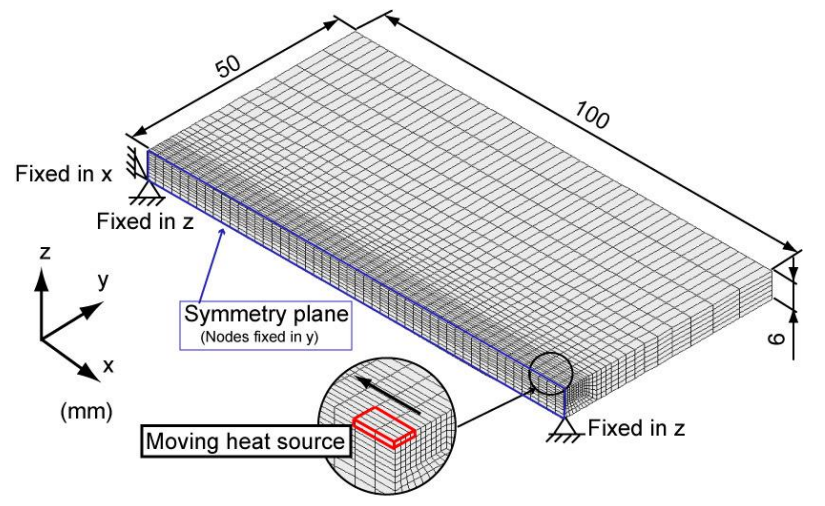

Fig. 3 Mesh division of welded plate model

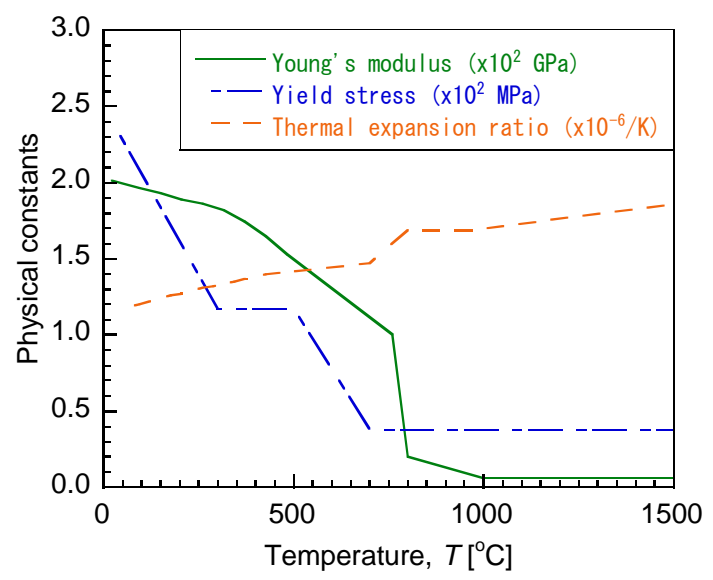

Fig. 4 Temperature dependent material constants of low alloy steel

low alloy steel used in nuclear reactor pressure vessels. Computations are done by Intel Xeon W5580.

\section{Comparison of the Computational Time}

The comparison of the computational time was carried out between ISM and the conventional method, which calculates the whole model as a strong nonlinear region, for three simple plate models composed of a varying number of elements. The computational time of the conventional method was too long, and so the total computation was extrapolated from the computational time in mid-flow.

Figure 5 represents the comparison of the computational time with each method. In the conventional method, the computational time increases with the increase in DOF. However, ISM shows a small increment of the computational time; in other words, the larger number of DOF means ISM has a larger advantage in computational time. The computational time of ISM for 282,000 DOF is approximately 24 hours, and the conventional method is approximately 1,300 hours. As can be seen, ISM is very advantageous and realistic for large-scale computations of welding problems.

\section{Comparison of the Residual Stress}

To verify the validity of ISM, the residual stress distribution is compared with that of the conventional method. In

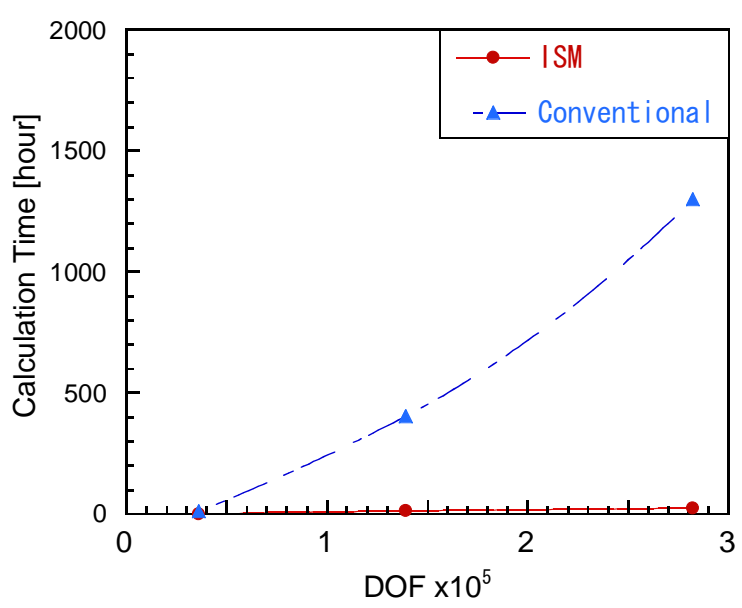

Fig. 5 Comparison of computational time between ISM and conventional method steel

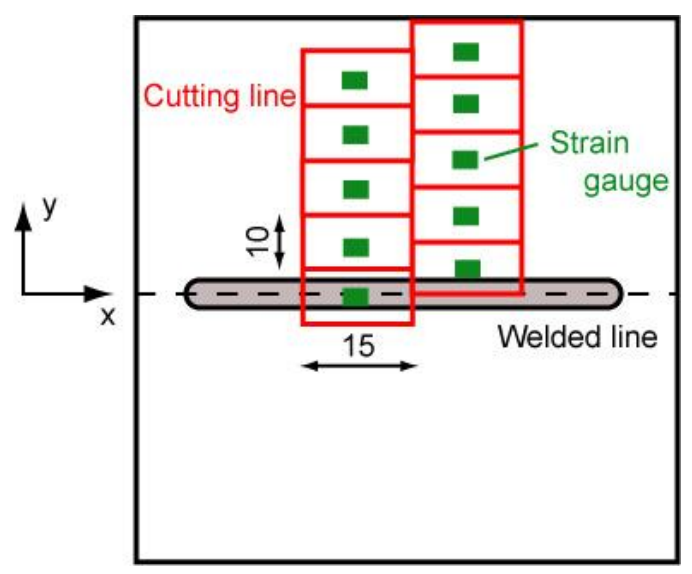

Fig. 6 Stress relaxation method

addition, a bead-on-plate welding experiment of low alloy steel plate in the same welding conditions was carried out. The residual stress in welding direction in the weld transverse distribution was measured in increments of $5 \mathrm{~mm}$ by the stress relaxation method. Strain gauges were fixed on the surface of the specimen after welding and cooling, and then the specimen was cut, as shown in Fig. 6.

The residual stress distribution in the welding direction from the welding line is shown in Fig. 7. The result of ISM agrees closely with the conventional method and experimental results. Especially, the maximum and the distribution, which are important factors of weld joint assessments, agree between the analysis and experiment.

\section{Comparison of the Angular Deformation}

The weldment changes shape by local heating and quenching. The welding simulation commonly performed for deformation assessment, which uses the angular deformation, was compared between each method.

Figure 8 represents the analysis result of the angular deformation. ISM agrees completely with the conventional method; that is, the analysis result by ISM has good prediction accuracy. From these results, it is seen that ISM does not affect on both of the residual stress and welding deformation. 


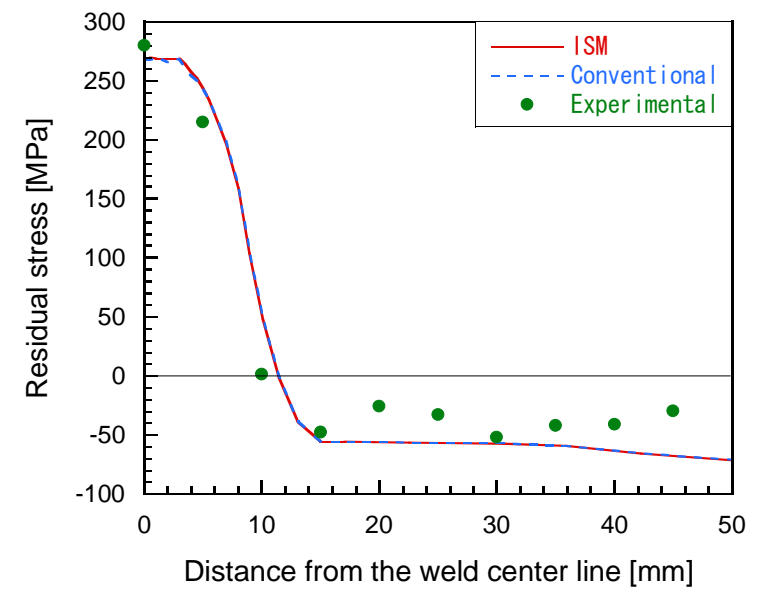

Fig. 7 Comparison of the residual stress in welding direction in transverse distribution between simulation and experiment

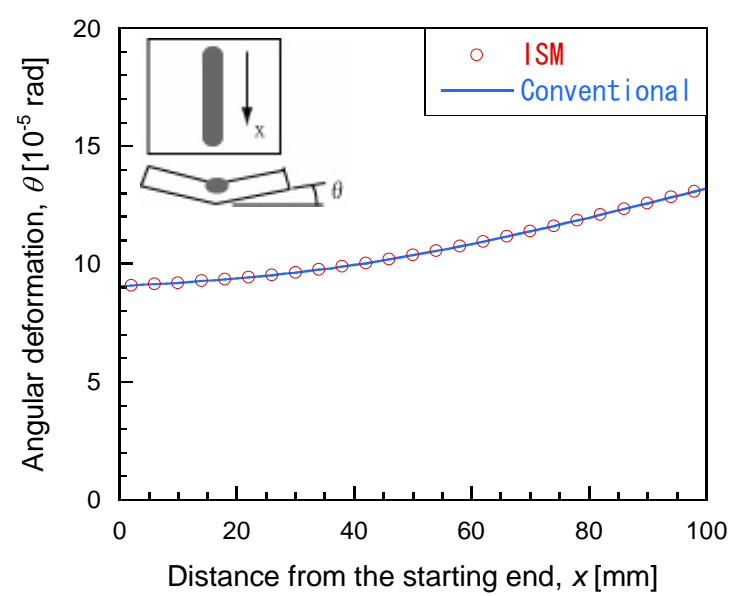

Fig. 8 Comparison of the angular deformation between ISM and conventional method

\section{Application of ISM to Actual Equipment}

\section{Background}

ISM was applied to actual equipment to determine its effectiveness. The damage case used as the analysis model was the pressurizer relief valve nozzle in Tsuruga Power Station Unit 2 in 2003, selected from the Nuclear Information Archives (NUCIA) released by the Japan Nuclear Technology Institute. $^{15)}$

The pressurizer relief valve nozzle consists of low alloy steel with a buttering weld of stainless steel on the inner surface. The multi-pass weld joint material is Ni based alloy 600 weld metal, jointed with stainless steel pipe (safe end) . In this case, SCC was generated in the Ni based alloy 600 weld metal due to the high tensile residual stress. Therefore, the model was created as shown in Figs. 9 and the residual stress after welding was examined. The model is a three-dimensional cylindrical shape with 28,908 elements and 95,688 DOF, and different properties were assigned to every element to represent the dissimilar weld joint. The locations of weld metal and the welding sequence are assumed as shown in Figs. 9(b). After the heat conduction analysis of the moving heat source, the stress field was

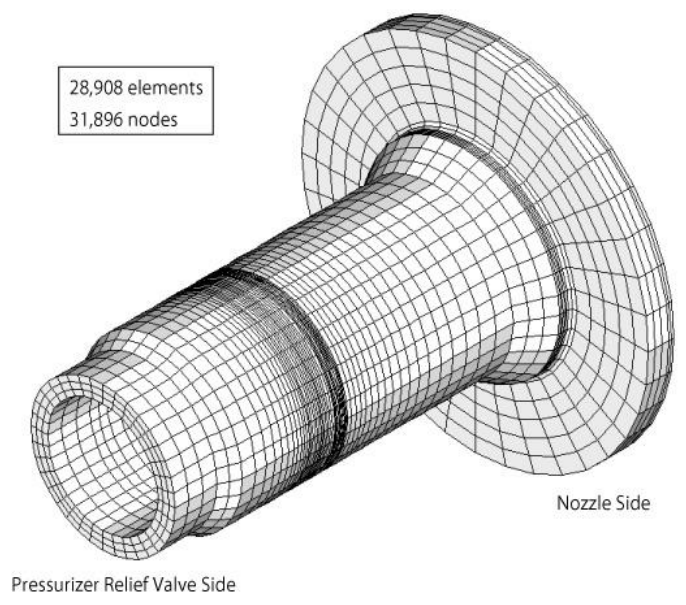

(a) Mesh division

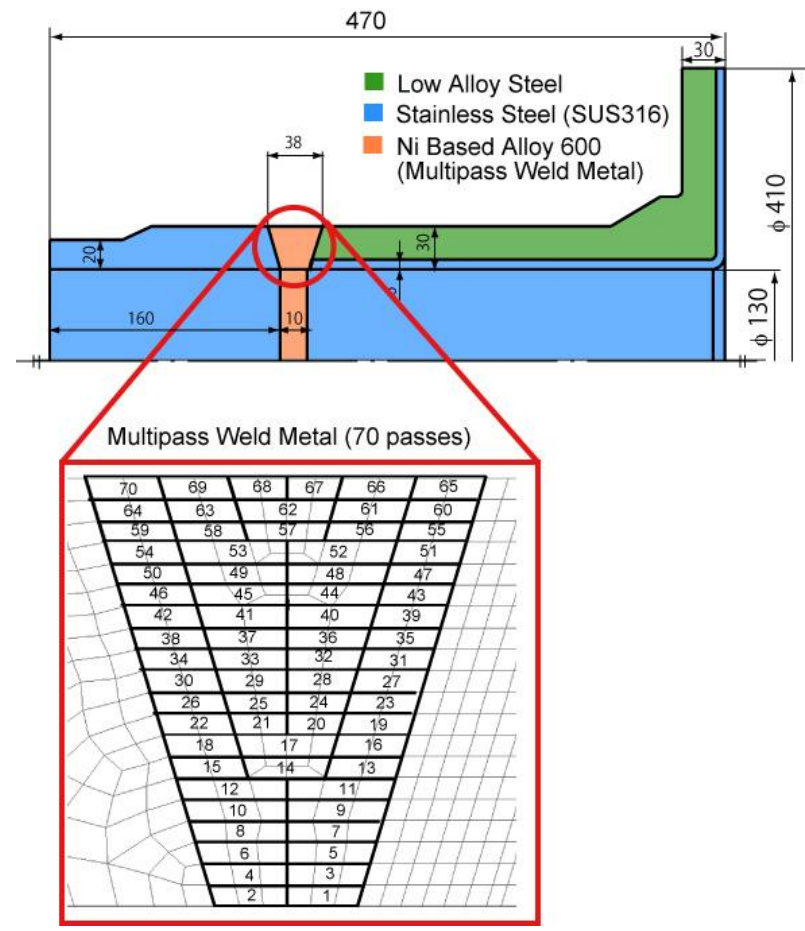

(b) Cross-sectional view of the model and multi-pass weld joint

Fig. 9 Pressurizer relief valve nozzle model

computed by ISM. The material properties of the low alloy steel, stainless steel and $\mathrm{Ni}$ based alloy have the temperature dependencies given in Fig. 3 and Figs. 10. The welding conditions were welding heat input $Q=1,200 \mathrm{~J} / \mathrm{deg}$, welding speed $v=1 \mathrm{deg} / \mathrm{s}$.

\section{Result and Discussion}

The contour figure of Mises stress distribution is shown in Fig. 11, the cross-sectional contour figures of the residual stress in the hoop, axial and radial directions of the central multi-pass joint of welding are shown in Figs. 12, and the cross-sectional contour figures in the welding start-end point after the multi-pass welding are shown in Figs. 13. As shown, high stress is caused by the multi-pass welding. The 


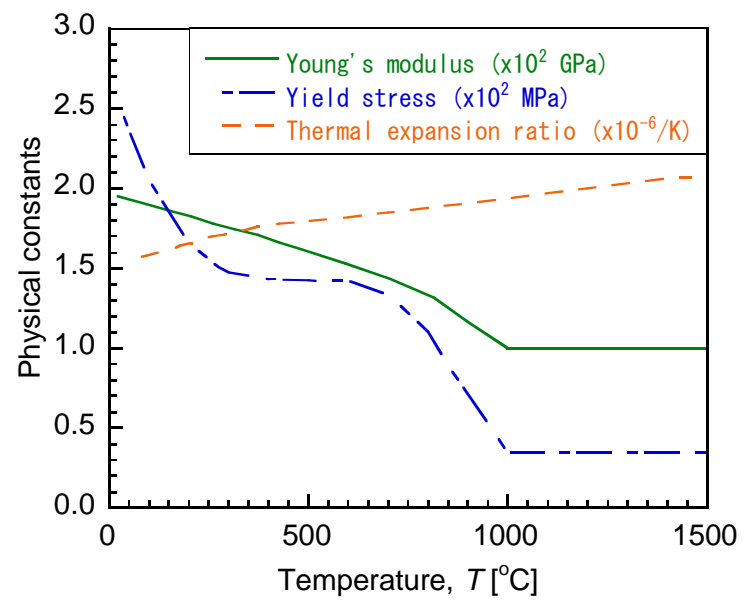

(a) Stainless steel

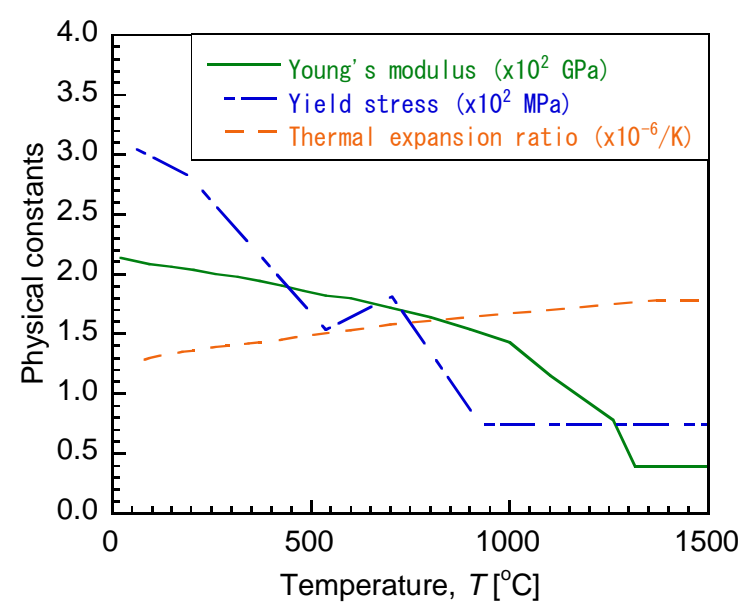

(b) Ni based alloy

Fig. 10 Temperature dependent material constants used in dissimilar welding analysis

stress in the radial direction is small because constraint condition in radial direction is free. High axial stress is present at the internal section of the joint but it is compressive on the inner surface. The weld joint area is constrained by the groove in axial direction, the residual stress is higher than in radial direction. The high tensile residual stress near the start-end point makes compressive ahead of start-end point in Figs. 12(b). There is large temperature difference in these two areas causes large stress difference. In the hoop direction, very high stress of more than $300 \mathrm{MPa}$ occurs at the inner section of the joint, and tensile stress reaching the yield stress of approximately $300 \mathrm{MPa}$ occurs on the inner surface because there is big constraint in the hoop direction by cylindrical configuration. There is compressive near the center of the multi-pass joint in Figs. 12(a), however the high tensile stress is occurred by the difference of the coefficient of thermal expansion between the stainless steel and the $\mathrm{Ni}$ based alloy at the stainless steel in Figs. 13(a). The coefficient of thermal expansion of the stainless steel is higher than the $\mathrm{Ni}$ based alloy, the stainless steel deforms bigger

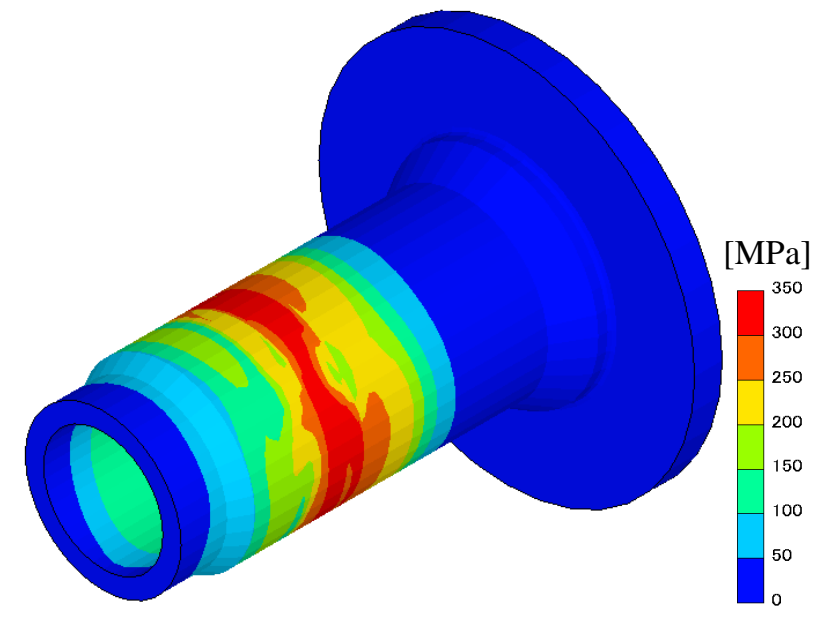

Fig. 11 Mises stress distribution after the welding

and high tensile stress is occurred in cooling process. Figure 14 shows the stress distribution on the inner surface. Especially, the residual stress in the hoop direction stress is elevated and reaches the yield stress. This condition of high tensile residual stress on the inner surface can cause SCC in many areas, including at the welding start-end point. Additionally, there is danger of cause SCC near the weld start-end point by high tensile stress.

The total computational time is approximately 384 hours (16 days), the total welding time is 21,600 seconds, and the total computational step is 135,942 steps. The computational time in the conventional method is approximately 115,200 hours (4,800 days) was extrapolated from the computational time in mid-flow.

In this paper shows that the high tensile stress is occurred near the weld start-end point and ISM is clearly beneficial as a large scale 3-dimensional analysis.

\section{Conclusion}

A large-scale weld residual stress analysis was applied to actual equipment, and the following results were obtained.

(1) The bead-on-plate weld analysis of the model with 282,000 DOF was carried out to verify the effect of reducing the computational time by ISM. ISM shortened the computational time to 24 hours from 1,300 hours in the comparison with the conventional method. The effect of the improved computing speed by ISM increases with the increase of the large-scale model.

(2) The comparison between ISM and the conventional method of the results of the residual stress bead-on-plate weld analysis was carried out, and the residual stress distribution was in agreement for each method and the experimental results. In addition, the angular deformation agreed between ISM and the conventional method.

(3) The multi-pass welding joint of the pressurizer relief valve nozzle was simulated to verify the possible application to actual equipment. The analysis was computed in realistic computational time. 


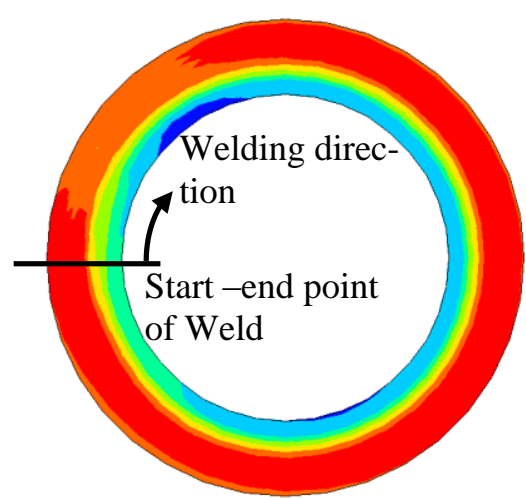

(a) Hoop direction

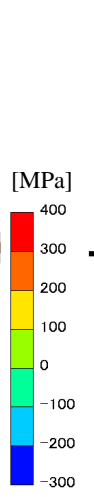

$-300$

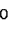

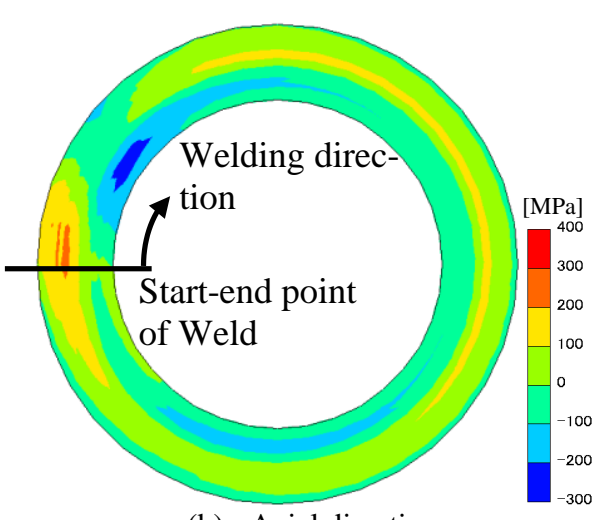

(b) Axial direction

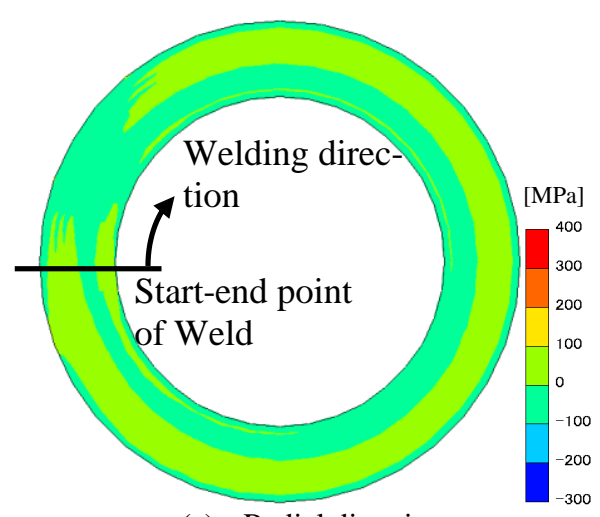

(c) Radial direction

Fig. 12 Cross-sectional view of the residual stress at the center of the multi-pass joint

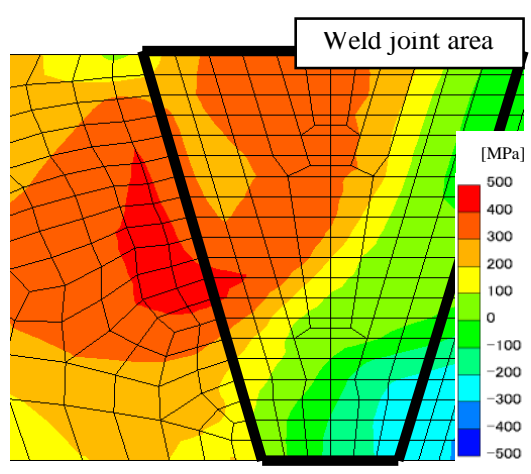

(a) Hoop direction

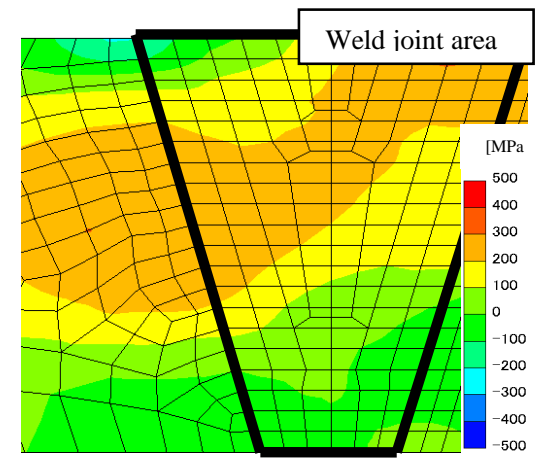

(b) Axial direction

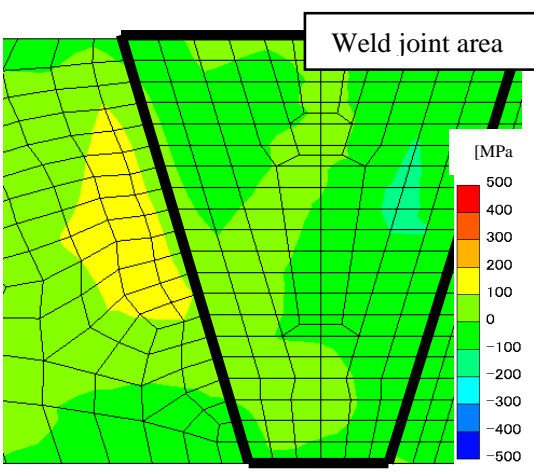

(c) Radial direction

Fig. 13 Cross-sectional view of the residual stress at the welding start-end point

\section{References}

1) S. Itoh, M. Shibahara, H. Serizawa, H. Murakawa, "Development of Hierarchical Multi-grid Method and Its Application to the Iterative Substructure Method," Proceedings of ISOPE'09, 700-705 (2009).

2) Y. Tian, C. Wanga, D. Zhub, Y. Zhouc, "Finite element modeling of electron beam welding of a large complex Al alloy structure by parallel computations," J. Mater. Process. Tech., 199 [1-3],41-48 (2007).

3) Y. Yogo, N. Iwata, K. Nakanishi, N. Goto, S. Yanagishita, "Development of the Fast FE Method for Welding Deformation (Report 1) : Development of the fast FE method and validation of the proposed method," J. Jpn. Weld. Soc., 78, 212-213 (2006), [in Japanese].

4) Y. Yogo, N. Iwata, K. Nakanishi, N. Goto, S. Yanagishita, "Development of the fast FE method for welding deformation (Report 2) : Accuracy validation of the proposed method by the side-rail model," J. Jpn. Weld. Soc., 78, 214-215 (2006), [in Japanese].

5) A. Kermanpur, M. Shamanian, V. Esfahani Yeganeh, "Three-dimensional thermal simulation and experimental investigation of GTAW circumferentially butt-welded Incoloy 800 pipes," J. Mater. Process. Tech., 199[1-3], 295-303 (2007).

6) M. Udagawa, J. Katsuyama, K. Onizawa, "Effects of welding methods and PWHT on residual stress distribution near the cladding layer of reactor pressure vessel," J. Jpn. Weld. Soc., 81, 4-5 (2007), [in Japanese].

7) H. Murakawa, I. Oda, S. Itoh, H. Serizawa, M. Shibahara, H.

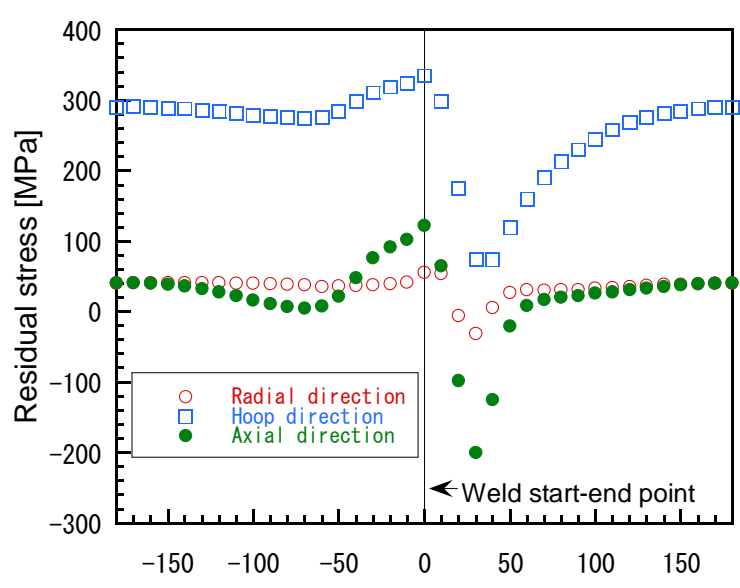

Fig. 14 Comparison of the residual stress

Nishikawa, "Iterative Substructure Method for fast computation of thermal elastic plastic welding problems," J. Kansai Soc. Naval Architects Jpn., 243, 67-70 (2005), [in Japanese].

8) H. Nishikawa, I. Oda, S. Itoh, M. Shibahara, H. Serizawa, H. Murakawa, "3-Dimensional High-Speed FEM for Thermal Elastic Plastic Welding Problems 4th German-Japanese Seminar - Materials," Processes and Components, University of Karlsruhe, Karlsruhe, Germany, Sept.7-8 (2005).

9) H. Murakawa, H. Serizawa, M. Shibahara, S. Itoh, "Fast 
Computational Scheme for Large Scale Nonlinear Transient Problems in Welding Mechanics," Proceedings of ICCES'05, Chennai, India, Dec. (2005).

10) H. Murakawa, I. Oda, S. Itoh, H. Serizawa, M. Shibahara, H. Nishikawa, "Iterative Substructure Method for fast FEM analysis of mechanical problems in welding," J. Jpn. Weld. Soc., 75, 274-275 (2004), [in Japanese].

11) H. Murakawa, I. Oda, S. Itoh, H. Serizawa, M. Shibahara, H. Nishikawa, "Iterative Substructure Method for fast computation of thermal elastic plastic welding problems," J. Kansai
Soc. Naval Architects Jpn., 23, 83-85 (2004), [in Japanese].

12) I. Oda, S. Itoh, M. Shiabahara, K. Masaoka, H. Serizawa, H. Murakawa, "FE analysis for deformation of multi-pass weld joint using Iterative Substructure Method (ISM)," J. Jpn. Weld. Soc., 77, 298-299 (2005), [in Japanese].

13) H. Nishikawa, H. Serizawa, H. Murakawa, "Actual application of large-scaled FEM for analysis of mechanical problems in welding," Q. J. Jpn. Weld. Soc., 24 [2], 168-173 (2006), [in Japanese].

14) NUCIA, http://www.nucia.jp/ 